\title{
UN MODELO DE SIMULACIÓN DE SISTEMAS DE ENGORDA DE BOVINOS A PASTOREO
}

\author{
Beef fattening simulation model for different grazing systems
}

\author{
Giorgio Castellaro G. ${ }^{1}$ *, Germán Klee G. ${ }^{2}$ y Jorge Chavarría R. ${ }^{3}$
}

\begin{abstract}
A B S T R A C T
A simulation model of beef cattle fattening systems based on grazing pastures was developed. The objective was to evaluate different pasture options under different management strategies, types of beef cattle, stocking densities, supplementation strategies and input prices. This model was structured based on a program, whose main module drives management input and generates result files. The dynamic section uses biological subroutines to solve equations simulating biological processes, the most important being the metabolizable energy intake and liveweight change. Pasture growth and nutritive value were simulated empirically from the interpolation of monthly data of pasture growth and digestibility of different pasture types. The model also calculates processes related to pasture management, forage conservation and bioeconomic aspects. The physical grazing experiment, carried out during the 1979-1980 seasons was utilized to validate the animal subsystem, A coefficient of determination higher than $95 \%$ was obtained when simulated and real values measured in the respective experiments were correlated by regression analysis, verifying the non existence of bias in the estimations. The root mean square error (RMSE) was smaller than $6 \%$, suggesting that this model is realistic and has a high degree of precision.
\end{abstract}

Key words: production systems, simulation model, subterranean clover.

\section{R E S U M E N}

Se elaboró un modelo de simulación de sistemas de producción de carne basado en utilización de pasturas, cuyo objetivo fue evaluar diferentes alternativas pratenses bajo distintas modalidades de manejo, tipos de bovinos, densidades de carga, estrategias de suplementación y precios de insumos. El modelo se estructuró sobre la base de un programa computacional, cuyo módulo principal maneja archivos de datos de manejo y genera archivos de resultados. La sección dinámica está estructurada en subrutinas orientadas a resolver ecuaciones que simulan los procesos biológicos, siendo los más importantes el consumo de energía metabolizable y el cambio de peso vivo. El crecimiento y valor nutritivo de los pastizales fue simulado empíricamente a partir de la interpolación de datos mensuales de tasas de crecimiento y digestibilidad de diferentes tipo de pasturas. El modelo también calcula los procesos relacionados con el manejo del pastoreo, conservación de forrajes y aspectos bioeconómicos. Para la validación del subsistema animal, se utilizaron los datos de peso vivo de toretes Hereford obtenidos de los experimentos físicos de pastoreo realizados durante la temporada 19791980. Al relacionar mediante un análisis de regresión los valores simulados con los medidos en los experimentos, se obtuvo un coeficiente de determinación superior al 95\%, confirmándose la inexistencia de sesgo en la estimación. La raíz del error cuadrático medio (RMSE) no superó el 6\%, lo que indicaría que este modelo es realista y tiene un alto grado de precisión.

Palabras clave: sistemas de producción, modelos de simulación, trébol subterráneo.

\footnotetext{
${ }^{1}$ Universidad de Chile, Facultad de Ciencias Agronómicas, Casilla 1004, Santiago de Chile. E-mail: gicastel@uchile.cl *Autor para correspondencia.

${ }^{2}$ Instituto de Investigaciones Agropecuarias, Centro Regional de Investigación Quilamapu, Casilla 426, Chillán, Chile. Recibido: 20 de enero de 2006. Aceptado: 16 de mayo de 2006.
} 


\section{INTRODUCCIÓN}

En la precordillera andina de la VIII Región de Chile existe una superficie de pastizales estimada en unas 190.000 ha, cuyo uso habitual ha sido la ganadería extensiva en combinación con cultivos tradicionales. La productividad anual de estos sistemas pecuarios no supera los 30 a $40 \mathrm{~kg}$ de peso vivo por hectárea (Klee et al., 1984a). Ante esta realidad, el Instituto de Investigaciones Agropecuarias (INIA) ha planteado sistemas de producción alternativos, sobre la base de métodos de pastoreo conservacionista, considerando la estacionalidad y producción de los pastizales, como también mediante la intensificación de los sistemas a través del establecimiento de pasturas no tradicionales para la zona, utilizadas mediante pastoreo con razas de bovinos especializadas (Klee y Chavarría, 1998). Lo anterior se ha estudiado mediante módulos de producción localizados en ambientes ecológicos representativos del área. Como complemento de este enfoque, se ha pensado en desarrollar un modelo de simulación de estos sistemas con el propósito de integrar en forma más ordenada los conocimientos acerca del funcionamiento de éstos, para ser utilizado en la evaluación de diferentes estrategias de manejo.

El objetivo principal de este trabajo fue la elaboración de un modelo de simulación de engorda de bovinos a pastoreo, y validar su comportamiento mediante la comparación de los valores simulados de peso vivo con los obtenidos en sistemas físicos de producción, basados en el uso de pasturas de trébol subterráneo (Trifolium subterraneum L.) utilizadas mediante pastoreo con toretes Hereford, sometidos a diferentes estrategias de suplementación.

\section{MATERIALES Y MÉTODOS}

El modelo se estructuró sobre la base de un programa computacional escrito en lenguaje Visual Basic Excel® versión 7.1, el cual está dividido en varios subprogramas que se describen a continuación:

\section{Programa principal}

Este programa maneja los archivos de entrada (información de manejo del predio y base de datos) y salida (resultados). En su sección dinámica maneja las subrutinas biológicas y de manejo y actualiza los niveles de peso vivo (PV), edad de los animales $\mathrm{y}$ fitomasa en cada uno de los potreros del predio simulado, mediante un procedimiento de integración numérica, considerando un intervalo de integración diario.

\section{Archivo de datos de manejo del predio}

En este archivo, se definen los datos de manejo del sistema a simular. El mismo está formado de varias secciones que se describen brevemente a continuación:

Manejo del predio. Incluye la información referida a la cantidad de potreros, y para cada uno de ellos se define la superficie, el valor monetario por hectárea, el grado de pendiente, el tipo de pastizal o pastura, la fitomasa inicial, la fecha de inicio y duración del período de rezago, el valor crítico de MS para efectuar un cambio de potrero, y el número mínimo de días de permanencia de los animales en un potrero.

Manejo de los animales. Esta sección contiene información respecto del número y tipo de animales, edad inicial, peso inicial, peso máximo alcanzado por los animales al momento de iniciar la simulación, fecha de ingreso a la engorda, criterio de término (peso o edad) y el peso o la edad de término deseado en los animales.

Manejo de la suplementación. Esta sección define el número de períodos de suplementación y, en cada uno de éstos, la fecha de inicio y duración de la suplementación, tipo y cantidad de suplemento ofrecido.

\section{Bases de datos (BD)}

Éstas proporcionan la información base respecto de parámetros genéticos de diferentes tipos de bovinos, información fisiológica, de calidad nutritiva y costos asociados a diferentes praderas y pasturas, como también información nutricional y de precios de distintos suplementos. Estos archivos son los siguientes:

Animales. Esta BD contiene la información de las clases de animales con que el modelo puede operar. Se definen el código del animal, especie, raza y tipo de bovino. También se incluyen en cada registro, una serie de coeficientes genéticos relacionados con las ecuaciones que calculan los requerimientos de mantención, el crecimiento potencial del animal y el valor energético del cambio de peso. 
Suplementos. Esta BD contiene datos con información referida a los diferentes tipos de suplementos. Cada línea define el código del suplemento, su información nutricional (porcentaje de MS, digestibilidad, concentración de energía metabolizable (EM), porcentaje de proteína cruda) y el precio por kilogramo de MS.

Praderas. Cada registro de esta BD contiene información referida a las diferentes praderas y pasturas. Se define el código del tipo de pastizal, el nombre, un factor de sensibilidad del consumo respecto de la digestibilidad del pastizal o pastura, el porcentaje promedio de leguminosas que hay en la misma, la distribución relativa mensual del crecimiento, la producción anual de fitomasa, los valores promedio mensuales de digestibilidad de la MS, el costo anual de establecimiento y mantención del pastizal y su duración en años.

Precios y costos unitarios. Esta BD contiene la información referida a la tasa de interés bancario mensual, los precios de compra y venta del kilogramo de carne bovina, los porcentajes de comisión de la feria para las transacciones de compra y venta de ganado, los costos unitarios de flete, vacunaciones, desparasitaciones, atención veterinaria y los costos de la jornada-hombre y hora-tractor.

\section{Subrutinas}

Estos subprogramas resuelven las ecuaciones que simulan los procesos biológicos en los cuales se basa el modelo, siendo el consumo de MS y el cambio de PV los fundamentales. En la primera versión de este modelo se supone como hipótesis principal que el factor nutricional más importante que limita la ganancia de peso de los bovinos es el consumo de energía metabolizable. Las subrutinas también calculan ciertos procesos relacionados con el manejo del pastoreo.

Subrutina consumo. Este subprograma determina el consumo de MS y EM efectuado por los animales en pastoreo. Estas variables son determinadas en función de variables relacionadas con el tipo de animal, disponibilidad de forraje y valor nutricional del pastizal. El consumo potencial (CONP) se calculó de acuerdo a lo propuesto por el Standing Committee on Agriculture, Ruminants Subcommittee (SCA, 1990):

$$
C O N P=J \cdot S R W \cdot W_{\text {rel }} \cdot\left(1,7-W_{\text {rel }}\right) \quad \text { (Ecuación 1) }
$$

donde $S R W$ es PV estándar a la madurez $(\mathrm{kg}), W_{\text {rel }}$ es el tamaño relativo (definido como la relación entre el PV normal y el peso estándar a la madurez (Ecuación 2)) y la constante $J$, la cual representa el consumo por unidad de peso vivo, tiene un valor de 0,024 para el caso de bovinos.

$$
W_{\text {rel }}=\frac{W_{\text {normal }}}{S R W}
$$

(Ecuación 2)

En la ecuación anterior, $W_{\text {normal }}$ es definido como el valor mínimo entre el PV máximo que el animal haya alcanzado en su vida $\left(W_{\max }, \mathrm{kg}\right)$ y el PV que el animal debiera tener según su edad (PQDT, kg):

$$
\left.W_{\text {normal }}=\operatorname{Mínimo~}\left(W_{\max }, P Q D T\right) \quad \text { (Ecuación } 3\right)
$$

PQDT está definida en función de la edad del animal (edad, días), de acuerdo a la siguiente ecuación (Brody, 1945):

$$
P Q D T=S R W-\left(S R W-S R W_{\text {nac }}\right) \times e^{-C_{\text {gro }} \text { edad }^{2}}
$$

\section{(Ecuación 4)}

donde $S R W_{n a c}$ es el peso estándar al nacimiento $(\mathrm{kg})$ y $C_{g r o}$ es un coeficiente que representa la velocidad de crecimiento del animal. El coeficiente anterior tiene un valor de 0,00158629 y se calculó a partir de los datos de crecimiento de bovinos proporcionados por García (1992).

El CONP se ajusta por factores asociados a la disponibilidad ( $F D I S P)$ de MS existente en el potrero que es pastoreado (Disp, $\mathrm{kg} \mathrm{ha}^{-1}$ ) y la digestibilidad $(F D I G)$ de la MS del forraje consumido $\left(D I G_{f c} \%\right)$, los cuales están formulados como multiplicadores:

$F D I S P=1-e^{-C f d \cdot D i s p}$

(Ecuación 5)

$F D I G=1-H \cdot\left(0,8-0,001 \cdot D I G_{f c}\right)+0,17 \cdot G$

(Ecuación 6)

En la ecuación 5, Cfd es un coeficiente que indica el grado de curvatura de la curva y representa una medida del cambio unitario que se produce en el consumo relativo debido al cambio en una unidad de disponibilidad de MS. Para el caso de bovinos, este coeficiente tiene un valor igual a 0,001664 
(Abreu, 1975). A su vez, en la ecuación 6, el coeficiente $H$ toma un valor igual a 1,7 para el caso de pasturas de gramíneas, y es igual a 1,0 para pastizales naturales. En la misma ecuación, $G$ representa la proporción promedio de leguminosas presentes en el pastizal (SCA, 1990).

De este modo, el consumo real de MS (CONR, $\left.\mathrm{kg} \mathrm{d}^{-1}\right)$ queda definido por la siguiente ecuación:

$$
C O N R=C O N P \cdot F D I S P \cdot F D I G \quad(\text { Ecuación 7) }
$$

Consumo potencial de suplementos. El modelo supone que el consumo potencial de suplementos $\left(C_{N} O N P_{s u p^{\prime}} \mathrm{kg} \mathrm{d}^{-1}\right)$ está regulado principalmente por el PV del animal $(W, \mathrm{~kg})$ y la calidad del recurso suplementario, medido a través de la metabolicidad $\left(Q_{m s}\right)$, utilizándose diferentes ecuaciones cuando el suplemento corresponde a forrajes o concentrados energéticos. Las ecuaciones utilizadas son las propuestas por el Agricultural and Food Research Council (AFRC) (Poppi, 1996):

$$
\operatorname{CONP}_{\text {sup }}=0,001 \cdot\left(116,8-46,6 \cdot Q_{m s}\right) \cdot W^{0,75}
$$

para concentrados

(Ecuación 8.1)

$$
\operatorname{CONP}_{\text {sup }}=0,001 \cdot\left(106,5 \cdot Q_{m s}+24,1\right) \cdot W^{0,75},
$$

para forrajes

(Ecuación 8.2)

La decisión de utilizar una u otra ecuación, se efectúa según el porcentaje de MS y la concentración de EM presente en la MS del suplemento $\left(M D_{s}\right.$, MJ $\mathrm{kg}^{-1}$ ), definiendo como concentrado a aquellos suplementos que presentan un porcentaje de MS superior o igual a $88 \%$ y un valor de $M D_{s}$ superior o igual a $11 \mathrm{MJ} \mathrm{kg}^{-1}$.

El CONP ${ }_{\text {sup }}$ se compara con la cantidad diaria ofrecida, utilizándose esta última cifra cuando $C O N P_{\text {sup }}$ supera a dicho valor.

Tasa de sustitución. Relacionado con el consumo de suplementos se considera además, el efecto sustitución $\left(T_{\text {sust }} \mathrm{kg} \mathrm{kg}^{-1}\right)$, el cual se define como la cantidad de MS de forraje proveniente del pastizal que se deja de consumir por cada kilogramo de MS de suplemento consumido (SCA, 1990). Se supone que existe sustitución de forraje cuando la digestibilidad del suplemento es mayor que la digestibili- dad del forraje proveniente del pastizal. Las variables determinantes de este efecto son la digestibilidad del forraje consumido proveniente del pastizal $\left(D I G_{f c}, \%\right)$, la digestibilidad del suplemento $\left(D I G_{s}\right.$, $\%$ ) y la disponibilidad de MS presente en el pastizal (Disp, $\left.\mathrm{kg} \mathrm{ha}^{-1}\right)$ La expresión matemática de esta hipótesis se calculó sobre la base de los datos proporcionados por SCA (1990):

Tsust $=\left(\frac{D I G_{f c}}{D I G_{S}}\right) \cdot\left(1-e^{-0,0017287 \cdot D i s p}\right) \quad$ (Ecuación 9)

Una vez calculada $T$ sust, se procede a corregir el consumo de MS proveniente del pastizal (CONR, $\mathrm{kg}$ $\left.\mathrm{d}^{-1}\right)$, definiendo de esta manera el consumo real de forraje proveniente del mismo (CONR prad $\left.\mathrm{kg} \mathrm{d}^{-1}\right)$ :

$$
\text { CONR }_{\text {prad }}=C O N R-T_{\text {sust }} \cdot C O N R_{\text {sup }}
$$

(Ecuación 10)

Cuando no existe sustitución $\left(D I G_{f c}>D I G_{s}\right)$, el consumo de pastizal no sufre modificaciones. En este caso, el consumo de suplementos se calcula como el valor mínimo entre el consumo potencial de suplementos definido por las ecuaciones 8.1 ú 8.2 y la diferencia entre el consumo potencial y el consumo de pastizal (CONP - CONR), suponiendo un efecto de complementariedad. Finalmente, se calcula la cantidad total de MS ingerida diariamente, a través de la suma del consumo real de pastizal y el consumo de suplemento, cifra que es acotada a un máximo equivalente al consumo potencial.

Subrutina cambio de peso. El cambio de peso en los animales se calcula en función de un balance entre los requerimientos energéticos de mantención y el consumo de EM. En el cálculo de los requerimientos de energía de mantención $\left(R M, \mathrm{MJ} \mathrm{d}^{-1}\right) \mathrm{se}$ usaron los estándares nutricionales proporcionados por SCA (1990):

$$
R M=\frac{K \cdot S \cdot 0,26 \cdot W^{0,75} \cdot e^{-0,03 \cdot A}}{K_{m}}+0,09 \cdot M E I+\frac{\text { Egrase }}{K_{m}}
$$

(Ecuación 11)

donde $K$ es un factor que depende de la especie animal (1,2 para Bos indicus; 1,4 para Bos taurus), $S$ es un factor de ajuste por sexo (1,00 para hembras y novillos; 1,15 para machos enteros), $W$ es el PV $(\mathrm{kg})$ del animal y $A$ es la edad expresada en años, con un valor máximo de 6 (cuando $\left.e^{(-0.03 \cdot A)}=0,84\right)$. 
En la ecuación anterior, $K_{m}$ es la eficiencia de utilización de la energía en el proceso de mantención, la cual depende de la concentración de EM de la dieta $\left(M D_{\text {diet }}, \mathrm{MJ} \mathrm{kg}^{-1}\right)$ :

$$
K_{m}=0,02 \cdot M D_{\text {diet }}+0,5
$$

(Ecuación 12)

La variable $M E I$ representa el consumo total de energía ( $\left.\mathrm{MJ} \mathrm{d}^{-1}\right)$, calculado en función del consumo total de MS (forraje del pastizal más el consumo de suplemento) y la concentración energética de la dieta $\left(M D_{\text {diet }}, \mathrm{MJ} \mathrm{kg}^{-1}\right)$ :

$M E I=C O N R \cdot M D_{\text {diet }}$

(Ecuación 13)

La variable $M D_{\text {diet }}$ se obtuvo mediante un promedio ponderado entre la concentración de EM del forraje consumido del pastizal $\left(M D_{\text {prac }}, \mathrm{MJ} \mathrm{kg}^{-1}\right) \mathrm{y}$ la del suplemento $\left(M D_{s}, \mathrm{MJ} \mathrm{kg}^{-1}\right)$, considerando sus respectivas proporciones en la dieta. $M D_{\text {prad }}$ y $M D_{s}$, se estimaron a partir de la digestibilidad de la MS del forraje consumido $\left(D I G_{f c}, \%\right)$ y la digestibilidad del suplemento ofrecido $\left(D I G_{s}, \%\right)$, respectivamente, según la ecuación propuesta por SCA (1990):

$M D=0,17 \cdot D I G-2$

(Ecuación 14)

La $D I G_{s}$ es un dato proporcionado por la base de datos respectiva, que almacena análisis bromatológicos de suplementos de uso común en rumiantes (NRC, 1985; AFRC, 1993). No obstante, para estimar la $D I G_{f c}$, se utilizó un índice de selección calculado a partir de los datos proporcionados por Jamieson y Hodgson (1979). Mediante este índice se corrige la digestibilidad del pastizal (DIG prad $\%)$, de acuerdo a la siguiente ecuación:

Iselec $=1+S_{\max } \cdot S_{\text {dig }} \cdot S_{\text {disp }}$

(Ecuación 15)

En la ecuación anterior, $S_{\max }$ representa la máxima selección, que en bovinos se estimó en un 28\% (Jamieson y Hodgson, 1979). $S_{\text {dig }}$ y $S_{\text {disp }}$ son multiplicadores que ajustan el valor máximo de selectividad de acuerdo con la digestibilidad de la pradera y su disponibilidad, respectivamente.

Una vez calculado Iselec, la variable $D I G_{f c}$ queda definido como:

$D I G_{f c}=D I G_{\text {prad }} \cdot$ Iselec

(Ecuación 16)
El incremento en el requerimiento de energía neta para mantención $\left(\mathrm{MJ} \mathrm{d}^{-1}\right)$, producto del efecto de las condiciones asociadas al pastoreo, está representado por la variable Egraze la cual es una función compleja que depende del tipo de terreno y de la disponibilidad y digestibilidad de la MS del pastizal (SCA, 1990):

Egraze $=\left[\left(C \cdot D M I \cdot\left(0,9-D I G_{f c}\right)\right)+\left(\frac{0,05 \cdot T}{(\text { Fitop }+3)}\right)\right] \cdot W$

(Ecuación 17)

donde $C$ es un coeficiente cuyo valor es de 0,006 para bovinos, $D M I\left(\mathrm{~kg} \mathrm{~d}^{-1}\right)$ es el consumo de MS proveniente del pastizal, excluyendo el aporte de MS proporcionada por los suplementos, $D I G_{f c}$ es la digestibilidad de la MS del forraje consumido (fracción decimal), $T$ es un coeficiente que refleja el grado de ondulación del terreno, siendo igual a 1,0, para terrenos planos (pendiente menor a $10 \%$ ); 1,5 para terrenos ondulados (pendiente entre 11 y $30 \%$ ) y 2,0 para terrenos montañosos (pendiente mayor a $30 \%$ ) (Holechek, 1988). Fitop (t ha $\left.{ }^{-1}\right)$ es la disponibilidad de MS presente en el pastizal y $W(\mathrm{~kg})$ el PV de los animales.

El cambio de peso $\left(C P V, \mathrm{~kg} \mathrm{~d}^{-1}\right)$ se calcula en función de la retención neta de energía (o balance de energía) representado por la variable RETEN y el valor energético del cambio de peso (VEDP, $\mathrm{MJ} \mathrm{kg}^{-1}$ de PV vacío):

$$
C P V=\frac{R E T E N}{0,92 \cdot V E D P}
$$

(Ecuación 18)

La retención neta de energía neta se calcula restando de la EM total consumida, la correspondiente a la necesaria para satisfacer los requerimientos de mantención. El resultado se pondera por la eficiencia de utilización de la EM en este proceso:

$$
\text { RETEN }=(M E I-R M) \cdot K_{f} \quad \text { si }(M E I-R M) \geq 0
$$

(Ecuación 19)

donde $K_{f}$ es la eficiencia de uso de la EM para el proceso de ganancia de peso, y al igual que $K_{m}$ es función de la concentración de EM de la dieta $\left(M D_{\text {diet }}, \mathrm{MJ} \mathrm{kg}^{-1}\right)$ :

$K_{f}=0,042 \cdot M D_{\text {diet }}+0,006$

(Ecuación 20) 
Cuando el balance es negativo, RETEN se calcula mediante la siguiente ecuación:

RETEN $=\frac{(M E I-R M)}{E M R C_{m}} \cdot K_{m}$ si $(M E I-R M)<0$

(Ecuación 21)

donde $E M R C_{m}$ representa la eficiencia de movilización de reservas corporales para el proceso de mantención y es igual a $80 \%\left(E M R C_{m}=0,8\right)$.

En la ecuación 18, la variable $\operatorname{VEDP}\left(\mathrm{MJ} \mathrm{kg}^{-1}\right)$ representa el contenido de energía de la ganancia (o pérdida) de peso, expresada sobre la base de PV "vacío". VEDP es función del PV del animal ( $W$, $\mathrm{kg})$, del PV estándar a la madurez $(S R W, \mathrm{~kg})$ y de un factor de ajuste por ganancia o pérdida de peso $(R)$, variables que se relacionan a través de las siguientes ecuaciones:

$$
R=2 \cdot\left(\frac{N E C}{N e m}-1\right)
$$

(Ecuación 22)

donde $N E C$ representa el valor absoluto del valor de la retención de energía disponible para ganancia (o pérdida) de peso definida por las ecuaciones 19 y 21 y $\mathrm{Nem}$ son los requerimientos de mantención expresados en energía neta $\left(\mathrm{MJ} \mathrm{d}^{-1}\right)$.

Calculadas las variables anteriores, VEDP queda definido por:

$V E D P=(6,7+\mathrm{R})+\frac{\text { Coef }-R}{1+e^{-6 \cdot(P-0,4)}} \quad($ Ecuación 23)

donde la variable Coef es un coeficiente dependiente de la raza del bovino. Coef es igual a 20,3 para todas las razas de tamaño medio, incluyendo a las provenientes de la especie Bos indicus y sus cruzamientos con Bos taurus. Para las razas europeas continentales como Charolais, Chianina, Blonde de Aquitania, Maine Anjou y Simmental, Coef toma un valor de 16,5. En la ecuación 23, $P$ es el cociente entre el PV del animal y el peso estándar a la madurez $\left(P=W \cdot S R W^{-I}\right)$.

Subrutina praderas. En esta subrutina se calcula la tasa de crecimiento de los diferentes pastizales $\left(T C, \mathrm{~kg} \mathrm{ha}^{-1} \mathrm{~d}^{-1}\right)$, la tasa de pérdida de MS (tperd, $\mathrm{kg} \mathrm{ha}^{-1} \mathrm{~d}^{-1}$ ) y la digestibilidad de la MS disponible (Dig, \%). La TC se calculó utilizando los datos de distribución promedio mensual del crecimiento
$\left(T C_{r e l}\right)$, los cuales se expresaron a nivel diario mediante una subrutina de interpolación segmentaria (Chapra y Canale, 1999). El valor así obtenido se multiplicó por la producción de MS anual definida para cada pastizal $\left(\right.$ FITOP $\left._{\max }, \mathrm{kg} \mathrm{ha}^{-1} \mathrm{año}^{-1}\right)$ y se dividió por un factor que representa el número de días promedio de cada mes $(30,42)$ :

$$
T C=\frac{T C_{r e l} \cdot \text { FITOP }_{\max }}{30,42}
$$

Para la tasa de pérdida de MS debida a efectos ajenos a la defoliación, se estimó un valor fijo equivalente a un $0,3 \%$ diario. La digestibilidad de la MS disponible se obtuvo a partir de los valores promedio mensuales definidos para cada tipo de pastizal $y$, al igual que en el caso del crecimiento, el valor diario se calculó mediante un procedimiento de interpolación segmentaria (Chapra y Canale, 1999).

Subrutina cambio de potrero. Esta subrutina efectúa los cambios de potrero tomando en cuenta los criterios de manejo definidos. Asigna los animales al potrero que presente una máxima disponibilidad al momento de tomar la decisión de cambio. Para esto último, esta subrutina ordena los potreros de mayor a menor en función de su disponibilidad de MS.

Subrutina rentabilidad. Esta subrutina calcula los principales componentes de los costos variables de operación (expresados en $\$$ cabeza $^{-1}$ y en $\$$ ha $^{-1}$ ), tales como los costos de reposición de animales (incluidos las comisiones y fletes), costos sanitarios, costos de suplementación y utilización de pastizales, costos de la mano de obra asociada, costos de venta (incluidos las comisiones y fletes) e imprevistos. También se determina el margen bruto operativo (expresado en $\$$ cabeza $^{-1}$ y en $\$ h^{-1}$ ). Para lo anterior se utilizó el procedimiento desarrollado por González y Velasco (1991). Además, contempla el cálculo de un costo fijo de oportunidad de la inversión, aplicado al valor de la tierra y a la reposición de animales (González, J. y Velasco, R. 1999. Instituto de Investigaciones Agropecuarias (INIA), comunicación personal), considerando el período de pastoreo simulado por el modelo y una tasa mensual de interés bancario, de acuerdo a la siguiente ecuación:

CostoAlt $=$ ValorInv $\cdot\left((1+\text { Tibm })^{\text {perpast }}-1\right)$

(Ecuación 25) 
donde CostoAlt, representa el costo alternativo asociado a la reposición de animales y/o al valor de la tierra, ValorInv $\left(\$\right.$ cabeza $\left.^{-1}\right)$ representa el valor total de la inversión en animales y/o tierra $\left(\$\right.$ cabeza $\left.^{-1}\right)$, Tibm es la tasa mensual de interés bancario (fracción) y perpast es el período de pastoreo (expresado en meses). En esta misma subrutina se calculan algunos indicadores simples tales como la relación Beneficio-Costo, considerando el Ingreso Bruto con relación al costo total.

\section{Archivos de resultados}

En estos archivos se almacenan los principales resultados biológicos obtenidos en la simulación, tales como la disponibilidad de MS del potrero que es pastoreado, la evolución del PV, las variables determinantes del consumo de MS y las variables asociadas al balance energético y el cambio de PV de los animales. Además, se genera un archivo resumen que contiene la información biológica más relevante tal como son el PV inicial y final obtenido por los animales, el consumo total de forrajes y suplementos, la duración del período de pastoreo y la ganancia promedio de PV, entre otras. También se incluye un resumen de los costos, ingresos e indicadores de rentabilidad asociados al sistema de producción simulado.

\section{Validación del modelo}

La validación del modelo de simulación elaborado se refirió solamente al subsistema animal, a través del análisis de la evolución del PV de los bovinos, desde el momento del destete hasta el peso (o edad) de término. Para realizar este análisis, se utilizaron los datos obtenidos en los experimentos de campo realizados por Klee et al. (1984a; 1984b), usando los registros de PV de toretes Hereford, obtenidos durante la temporada 1979-1980. Para este tipo de animales se utilizaron los coeficientes genéticos correspondientes a bovinos de razas europeas $(S R W=770$ $\mathrm{kg}$; SRWnac $=35 \mathrm{~kg} ; K=1,40 ; S=1,15 ;$ Coef $=$ $20,3)$. Se simuló un predio de un solo potrero sembrado con un pastizal de trébol subterráneo, cuyas tasas de crecimiento y digestibilidades promedio mensuales, se presentan en el Cuadro 1.

El pastizal se pastoreó con una carga de 1,36 toretes $\mathrm{ha}^{-1}$ a partir del día 4 de abril. En dicho momento, el peso y edad promedio de los toretes fue de $192 \mathrm{~kg}$ y seis meses, respectivamente. La disponibilidad inicial de MS en el pastizal se estimó en $1.500 \mathrm{~kg} \mathrm{ha}^{-1}$. En la primera validación se contempló el uso de una suplementación con heno de trébol subterráneo ( $88 \%$
Cuadro 1. Tasa de crecimiento y digestibilidad de la materia seca (MS) de una pastura de trébol subterráneo de los ensayos de pastoreo, utilizados en la validación del modelo.

Table 1. Growth rate and dry matter (MS) digestibility of the subterranean clover pasture of the grazing trials utilized for the model validation.

\begin{tabular}{|c|c|c|}
\hline Mes & $\begin{array}{c}\text { Digestibilidad } \\
\text { de la } \mathrm{MS}^{1} \\
(\%)\end{array}$ & $\begin{array}{c}\text { Tasa de } \\
\text { crecimiento } \\
\left(\mathrm{kg} \mathrm{ha}^{-1} \mathrm{~d}^{-1}\right)\end{array}$ \\
\hline Enero & 57,0 & 0,0 \\
\hline Febrero & 52,0 & 0,0 \\
\hline Marzo & 50,0 & 2,4 \\
\hline Abril & 49,0 & 0,8 \\
\hline Mayo & 48,0 & 7,9 \\
\hline Junio & 50,0 & 22,9 \\
\hline Julio & 52,0 & 51,9 \\
\hline Agosto & 55,0 & 60,2 \\
\hline Septiembre & 62,0 & 37,5 \\
\hline Octubre & 69,0 & 14,0 \\
\hline Noviembre & 66,0 & 0,0 \\
\hline Diciembre & 59,0 & 0,0 \\
\hline
\end{tabular}

${ }^{1}$ Estimación propia de los autores.

2 Datos calculados a partir de una producción anual de MS de $6.000 \mathrm{~kg} \mathrm{ha}^{-1}$ (Klee et al., 1984a).

de MS; 60\% de digestibilidad; $8,2 \mathrm{MJ} \mathrm{kg}^{-1}$ de EM) a razón de 2,58 $\mathrm{kg}$ de MS por torete $\mathrm{d}^{-1}$, efectuada a partir del día 1 de julio, por un período de 92 días. En la segunda validación se utilizó el mismo pastizal, el mismo tipo de bovinos, de similar edad pero con diferente peso inicial, el cual fue de $169 \mathrm{~kg}$. En este caso, la suplementación consideró el uso de un suplemento simple constituido por una mezcla de concentrado basado en grano de avena (Avena sati$v a \mathrm{~L}$.) y heno de trébol subterráneo ( $88 \%$ de MS; $68,8 \%$ de digestibilidad; $9,7 \mathrm{MJ} \mathrm{kg}^{-1}$ de EM), el cual se ofreció a razón de $4,1 \mathrm{~kg}$ torete $\mathrm{d}^{-1}$, desde el 20 de abril, por un período de 163 días. En ambas situaciones se efectuó una simulación que duró 365 días a partir de la fecha de ingreso de los animales al pastizal, con el propósito de que al final del período de pastoreo simulado, los animales tuviesen una edad promedio de 18 meses.

La bondad de ajuste del modelo se evaluó a través del cálculo de mínimos cuadrados obtenidos para la regresión lineal $(Y=a+b \cdot X)$ calculada entre los valores observados $(\mathrm{X})$ y los valores simulados $(\mathrm{Y})$ (Shannon, 1988). Los coeficientes de estas ecuaciones se evaluaron planteando pruebas de hipótesis si- 
multáneas para el intercepto (Ho: $a=0$ ) y para la pendiente (Ho: $b=1)$ mediante la prueba " $\mathrm{t}$ " de student y un valor $\mathrm{P} \leq 0,05$ (Steel y Torrie, 1988). Adicionalmente, se calculó la raíz del error cuadrado medio de la predicción (RMSE), expresando el resultado tanto en unidades absolutas, como también en unidades porcentuales, con relación al valor promedio obtenido en las observaciones reales (Kabat et al., 1995). Este coeficiente indica el grado de sobre o subestimación que realiza el modelo respecto al promedio de los valores observados:

$$
R M S E=\sqrt{\frac{\sum\left(\text { ValSim }_{i}-\text { ValObs }_{i}\right)^{2}}{n}} \cdot \frac{100}{\text { ValObsProm }}
$$

(Ecuación 26)

En la ecuación anterior, ValSim $_{i}$ y ValObs $_{i}$ representan los valores de las observaciones simuladas y las medidas en terreno, respectivamente. ValObsProm es el promedio de las observaciones reales y $\mathrm{n}$ representa el número de pares de datos utilizados en el cálculo.

\section{RESULTADOS Y DISCUSIÓN}

\section{Validación 1. Toretes en pastoreo suplementa-} dos con heno. En la Figura 1a se presenta la evolución del PV de los toretes, con los valores reales y simulados correspondientes al primer experimento utilizado en la validación (Klee et al., 1984a).
A los 18 meses los toretes alcanzaron un peso de 344 $\mathrm{kg}$, siendo la ganancia promedio diaria por torete, entre los 6 y 18 meses, de $0,417 \mathrm{~g} \mathrm{~d}^{-1}$. La producción de PV del sistema fue de 207,2 $\mathrm{kg} \mathrm{ha}^{-1}$. Estos valores fueron similares a los medidos en las condiciones del experimento anteriormente citado $(356 \mathrm{~kg} ; 0,430 \mathrm{~kg}$ torete $^{-1} \mathrm{~d}^{-1}$ y $224 \mathrm{~kg} \mathrm{ha}^{-1}$, para el PV a los 18 meses, ganancia individual y producción de $\mathrm{PV}$ del sistema, respectivamente).

Al efectuar un análisis de regresión entre los valores reales y los simulados por el modelo, se determinó un coeficiente de determinación de $95 \%$. La recta de regresión calculada no difirió significativamente de la línea de bisectriz $(P>0,05)$, lo que indica que el modelo presenta un buen equilibrio, evidenciándose por lo tanto, la inexistencia de sesgo (Figura 1b). El valor de RMSE fue de 15,2 $\mathrm{kg}$ representando un $5,9 \%$ respecto del promedio de los valores reales.

Validación 2. Toretes en pastoreo suplementados con heno y grano de avena. El resultado de esta simulación, la cual incluye los valores de PV reales (Klee et al., 1984b) y simulados por el modelo, se puede apreciar en la Figura 2a.

En este caso, el modelo simuló $366 \mathrm{~kg}$ de PV para los toretes a los 18 meses de edad, siendo la ganancia promedio individual de los mismos, entre los 6 y los 18 meses, de $0,540 \mathrm{~kg} \mathrm{~d}^{-1}$. La producción de (a)

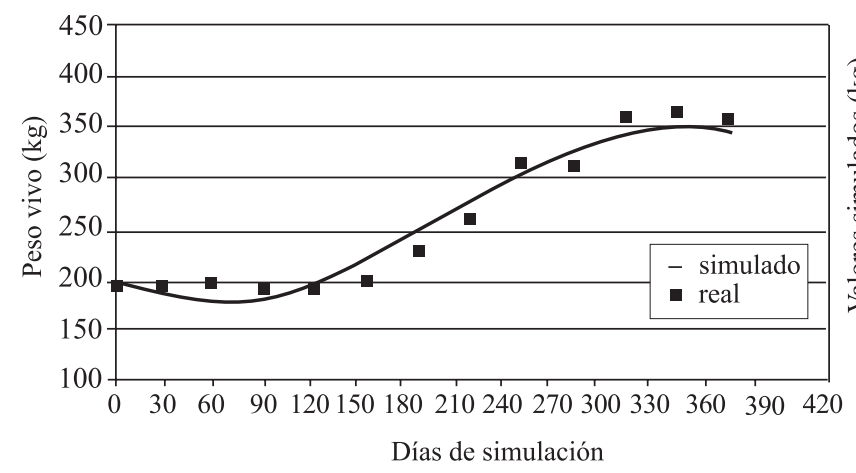

(b)

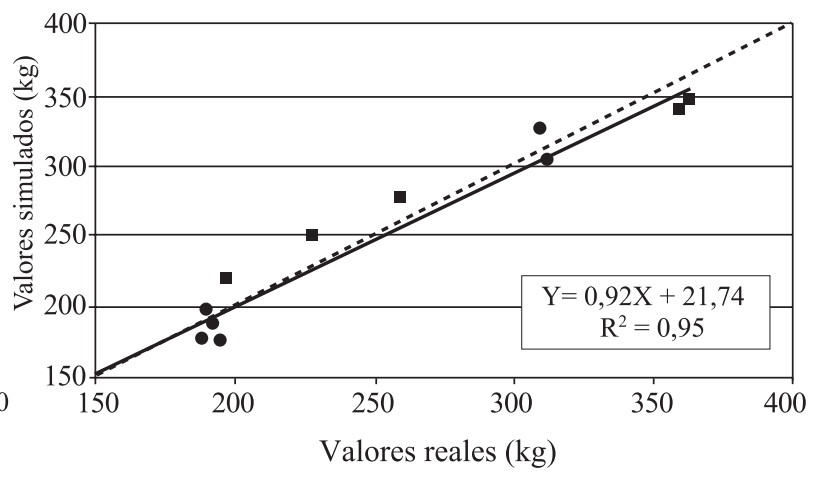

Figura 1. Variación del peso vivo de los toretes, desde el destete hasta los $360 \mathrm{~kg}$, pastoreando pastura de trébol subterráneo y recibiendo heno como suplemento: (a) Evolución en el tiempo. (b) Ecuación de regresión entre los valores simulados (Y) y los valores reales medidos en el experimento de pastoreo (X). La línea punteada indica la bisectriz $(\mathrm{Y}=\mathrm{X})$.

Figure 1. Bull calves live weight variations, since weaning until $360 \mathrm{~kg}$, grazing subterranean clover pasture and receiving hay as supplement. (a) Time evolution. (b) Regression equation between simulated live weight (Y) and real values measured in the grazing experiment $(X)$. The dotted line indicates the bisectrix $(Y=X)$. 
PV del sistema fue de $267,8 \mathrm{~kg} \mathrm{ha}^{-1}$. Al igual que en el caso anterior, estos valores fueron muy consistentes con los medidos en el experimento de campo $\left(357 \mathrm{~kg} ; 0,520 \mathrm{~kg}\right.$ torete $\mathrm{d}^{-1} \mathrm{~d}^{-1}$ y $268 \mathrm{~kg} \mathrm{ha}^{-1}$, para el PV a los 18 meses, ganancia individual y producción de PV del sistema, respectivamente).

El grado de ajuste entre los valores simulados y los determinados experimentalmente fue de 99\% (Figura 2b) y al igual que en la validación anterior, la recta de regresión calculada no difirió significativamente de la línea de bisectriz $(\mathrm{P}>0,05)$, lo que denota la inexistencia de sesgo en la estimación. En este caso, se obtuvo un valor de RMSE de $9,7 \mathrm{~kg}$, lo que representó un $3,6 \%$ respecto del promedio de los valores reales.

\section{Proyecciones futuras}

Los resultados anteriores confirman que este modelo, en su primera versión, simula en forma realista la evolución del PV de toretes Hereford desde la etapa de destete hasta la edad (o peso) de faenamiento, considerando al balance energético como el principal proceso. No obstante lo anterior, se sugiere incorporar aspectos relacionados con el efecto del contenido de proteína del forraje sobre el consumo y el cambio de peso, lo que podría ser importante, sobre todo en pastizales de clima mediterráneo, durante el período seco, donde probablemente este nutrimento pudiese ser limitativo para los procesos de consumo de MS y síntesis microbiana (Owens y Zinn, 1988; SCA, 1990).

(a)

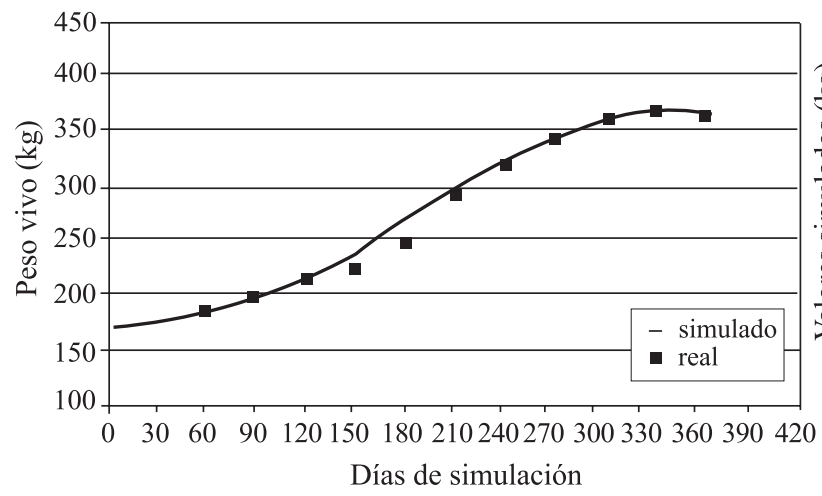

También resulta de interés, incorporar en este modelo una subrutina dinámica que de cuenta del crecimiento y el valor nutritivo de los diferentes tipos de pastizales, para proporcionar de este modo un mayor grado de realismo al modelo. Lo anterior permitiría por una parte, una independencia con respecto a la disponibilidad de datos de tasas de crecimiento y digestibilidad de diferentes recursos forrajeros para poder realizar simulaciones de diferentes alternativas de engorda. Por otro lado, al considerar la simulación del crecimiento y el valor nutricional de los pastizales en función de variables edafoclimáticas, se podrían realizar experimentos simulados de largo plazo, considerando los efectos de los cambios en las condiciones climáticas sobre dichas variables y por ende evaluar su efecto sobre el consumo y el comportamiento productivo de los animales. Bajo este enfoque, y al poder simular muchos años, se podrían obtener funciones de distribución de la producción de PV y de ese modo poder evaluar sistemas de producción incluyendo aspectos relacionados con la sustentabilidad y el riesgo asociado a diferentes estrategias de producción (Castellaro, 2003).

\section{CONCLUSIONES}

Es posible simular la evolución de los pesos vivos de toretes pastoreando pasturas de trébol subterráneo, así como evaluar el efecto de diferentes modalidades de suplementación sobre esta variable de estado. De

Figura 2. Variación del peso vivo de los toretes, desde el destete hasta los $360 \mathrm{~kg}$, pastoreando pastura de trébol subterráneo y recibiendo heno y avena-grano como suplemento: (a) Evolución en el tiempo. (b) Ecuación de regresión entre los valores simulados (Y) y los valores reales medidos en el experimento (X). La línea punteada indica la bisectriz $(Y=X)$.

Figure 2. Bull calves live weight variations, from weaning up to $360 \mathrm{~kg}$, grazing subterranean clover pasture and receiving hay and oat grain as supplement: (a) Time evolution. (b) Regression equation between simulated values $(Y)$ and real values measured in the grazing experiment $(X)$. The dotted line indicates the bisectrix $(Y=X)$. 
acuerdo con las pruebas estadísticas realizadas, el modelo presenta un alto grado de precisión ( $\geq 95 \%$ ), no observándose sesgo en las estimaciones.

De acuerdo con lo anterior, este modelo podría ser utilizado en la evaluación de sistemas de producción bovina, en su etapa de recría y engorda, en condiciones pastoriles.
Para dar un mayor realismo y versatilidad a este modelo de pastoreo, se sugiere incorporarle una subrutina dinámica de menor grado de empirismo orientada a simular el crecimiento y el valor nutritivo de los pastizales en función de variables climáticas y edáficas, como también mejorar los aspectos relacionados con el efecto del contenido proteico sobre el consumo y cambio de peso.

\section{LITERATURA CITADA}

Abreu, M.P. 1975. Simulación del crecimiento y performance reproductiva en vaquillonas de primero y segundo entore. p. 39-48. Informe de Conferencias. Cursos y Reuniones $\mathrm{N}^{\circ}$ 63. In $\mathrm{H}$. Caballero D. (ed.). Sistemas de producción pecuaria: principios y aplicaciones de investigación y extensión. Instituto Interamericano de Cooperación para la Agricultura (IICA), Montevideo. Uruguay.

AFRC. 1993. Necesidades energéticas y proteicas de los rumiantes. Apéndice I. Tabla de composición de alimentos. p. 147-157. Agricultural and Food Research Council (AFRC). Editorial Acribia, Zaragoza, España.

Brody, S. 1945. Bioenergetics and growth. 1.023 p. Reinhold Publishing Corporation, New York, USA.

Castellaro, G.G. 2003. Crecimiento de praderas mesofíticas a largo plazo, en respuesta a factores edafoclimáticos y modalidades de defoliación. 132 p. Tesis Magister en Ciencias Animales. Pontificia Universidad Católica de Chile, Facultad de Agronomía e Ingeniería Forestal, Santiago, Chile.

Chapra, S.C., y R.P. Canale. 1999. Métodos numéricos para ingenieros. $3^{\text {a }}$ ed. p. 502-536. Cap. 18. Interpolación. MacGraw-Hill, Santiago, Chile.

García, G.F. 1992. Requerimiento de proteína en el ganado lechero. p. 101-131. Capítulo II. In M. E. Ruiz (ed.) Simulación de sistemas pecuarios. Instituto Interamericano de Cooperación para la Agricultura (IICA), Red de Investigación en Sistemas de Producción Animal de Latinoamérica, San José, Costa Rica.

González, J., y R. Velasco. 1991. Evaluación económica en producción de carne bovina. Investigación y Progreso Agropecuario Quilamapu No 49 p. 3-11.

Holechek, J.L. 1988. An approach for setting the stocking rate. Rangeland 10:10-14.

Jamieson, W.S., and J. Hodgson. 1979. The effects of variation in sward characteristics upon the ingestive behaviour and herbage intake of calves and lambs under a continuous stocking management. Grass Forage Sci. 34:273-282.
Kabat, P.M., B.J. van den Broek, B. Marshall, J. Vos, and H. van Keulen. 1995. Modelling and parameterization of the soil - plant - atmosphere system. A comparison of potato growth models. 520 p. Wageningen Academic Publishers, Wageningen, The Netherlands.

Klee, G., y J. Chavarría, 1998. Engorda semi-intensiva de toritos Hereford en el secano de la precordillera andina de la VIII Región utilizando pastizales no tradicionales. p. 79-80. Libro de Resúmenes. XXIII Reunión Anual SOCHIPA A. G., Chillán, Chile. 2123 de octubre de 1998. Sociedad Chilena de Producción Animal (SOCHIPA), Chillán, Chile.

Klee, G., I. Ruiz, y H. Acuña. 1984a. Evaluación de sistemas de producción de carne en la precordillera de Bío Bío. I. Un sistema utilizando sólo trébol subterráneo como recurso alimenticio. Agric. Téc. (Chile) 44:27-38.

Klee, G., I. Ruiz, y H. Acuña. 1984b. Evaluación de sistemas de producción de carne en la precordillera de Bío Bío. II. Utilización de la pradera de trébol subterráneo mejorada y uso limitado de concentrado. Agric. Téc. (Chile) 44:45-54.

Owens, F.N., and R. Zinn. 1988. Protein metabolism of ruminant animals. p. 227-249. In D.C. Church (ed.). The ruminant animal. Digestive physiology and nutrition. Waveland Press, Long Grove, Illinois, USA.

NRC. 1984. Composition of feeds and mineral supplements. p. 47-63. In National Research Council (NRC). Nutrient requirements of beef cattle. $6^{\text {th }}$ ed. National Academic Press, Washington D.C., USA.

Poppi, D.P. 1996. Predictions of food intake in ruminants from analyses of food composition. Austr. J. Agric. Res. 47:489-504.

Shannon, E. 1988. Simulación de sistemas. Diseño, desarrollo e implantación. 427 p. Editorial Trillas, México DF.

SCA. 1990. Feeding standards for Australian livestock. 266 p. Standing Committee on Agriculture (SCA), Ruminants Subcommittee. CSIRO Publications, Melbourne, Australia.

Steel, R.G.D., y J.H. Torrie. 1988. Bioestadística. Principios y procedimientos. $622 \mathrm{p}$. McGraw-Hill, New York, USA. 